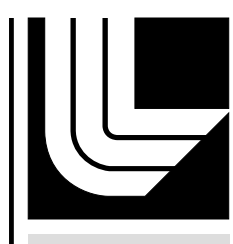

LA W RENCE LIVERMORE NATIONAL LABORATORY

Target Diagnostics Supports NIF's Path to Ignition

R. Shelton

December 15, 2011 
This document was prepared as an account of work sponsored by an agency of the United States government. Neither the United States government nor Lawrence Livermore National Security, LLC, nor any of their employees makes any warranty, expressed or implied, or assumes any legal liability or responsibility for the accuracy, completeness, or usefulness of any information, apparatus, product, or process disclosed, or represents that its use would not infringe privately owned rights. Reference herein to any specific commercial product, process, or service by trade name, trademark, manufacturer, or otherwise does not necessarily constitute or imply its endorsement, recommendation, or favoring by the United States government or Lawrence Livermore National Security, LLC. The views and opinions of authors expressed herein do not necessarily state or reflect those of the United States government or Lawrence Livermore National Security, LLC, and shall not be used for advertising or product endorsement purposes.

This work performed under the auspices of the U.S. Department of Energy by Lawrence Livermore National Laboratory under Contract DE-AC52-07NA27344. 


\section{X.XX Target Diagnostics Supports NIF's Path to Ignition}

The physics requirements derived from The National Ignition Facility (NIF) experimental campaigns are leading to a wide variety of target diagnostics. Software development for the control and analysis of these diagnostics is included in the NIF Integrated Computer Control System, Diagnostic Control System and Data Visualization. These projects implement the configuration, controls, data analysis and visual representation of most of these diagnostics. To date, over 40 target diagnostics have been developed to support NIF experiments.

In 2011 diagnostics were developed or enhanced to measure Ignition performance in a high neutron yield environment. Performance is optimized around four key variables: Adiabat $(\alpha)$ which is the strength and timing of four shocks delivered to the target, Velocity (V) of the imploding target, Mix (M) is the uniformity of the burn, and the Shape (S) of the imploding Deuterium Tritium (DT) hot spot. The diagnostics used to measure each of these parameters is shown in figure 1.

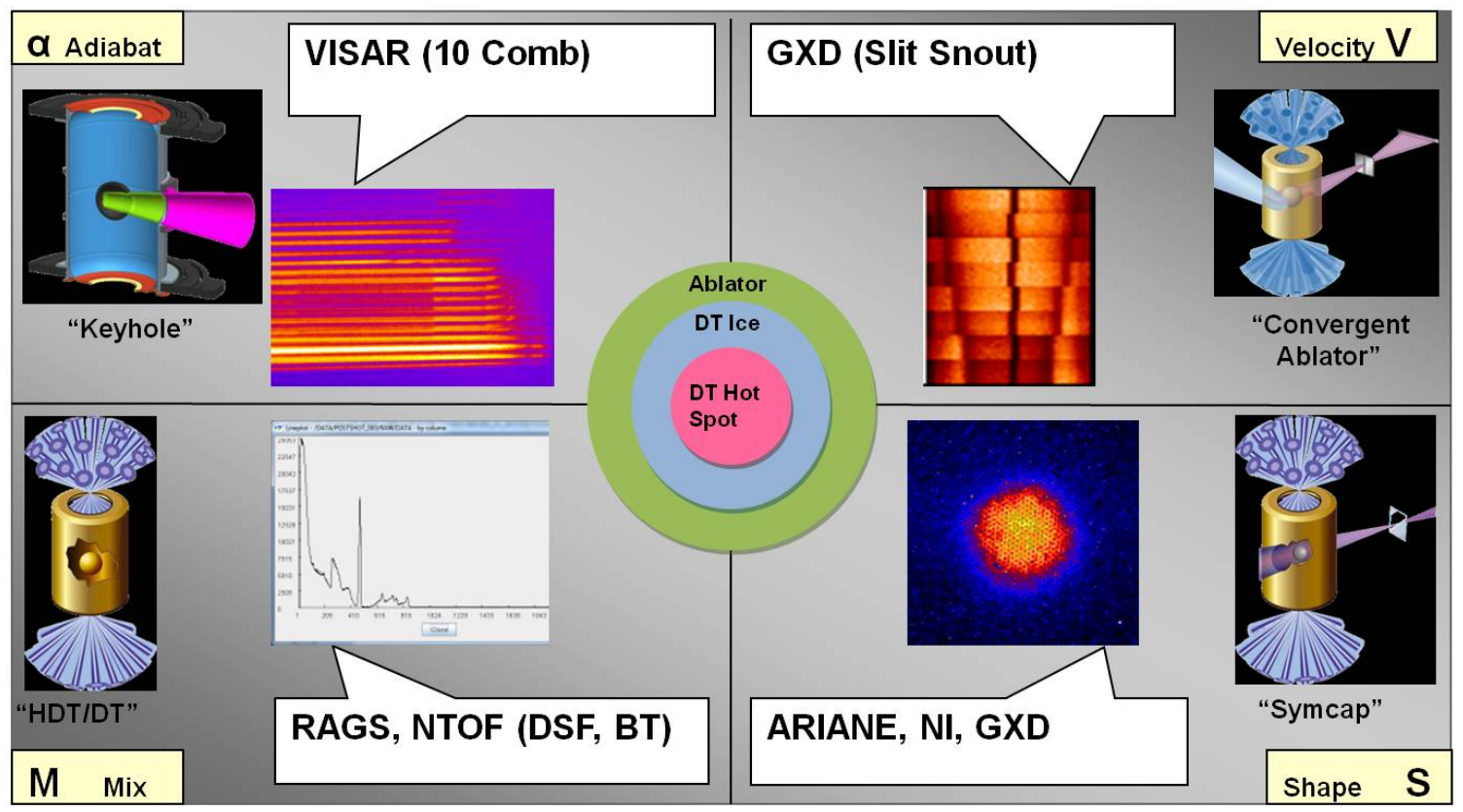

Figure 1. FY11 target diagnostics supporting ignition performance measurements 
Adiabat is measured using the Velocity Interferometer System for Any Reflector (VISAR) diagnostic consisting of three streak cameras. To provide for more accurate adiabat measurements the VISAR streak cameras were enhanced in FY11 with a ten comb fiducial signal controller to allow for post shot correction of the streak camera sweep non-linearity.

Mix is measured by the Neutron Time of Flight (NTOF) and Radiochemical Analysis of Gaseous Samples (RAGS) diagnostics. To accommodate high neutron yield shots, NTOF diagnostic controls are being modified to use Mach Zehnder interferometer signals to allow the digitizers to be moved from near the target chamber to the neutron shielded diagnostic mezzanine.

In December 2011 the first phase of RAGS diagnostic commissioning will be completed. This diagnostic will analyze the tracers that are added to NIF target capsules that undergo nuclear reactions during the shot. These gases are collected and purified for nuclear counting by the RAGS system. Three new instrument controllers were developed and commissioned to support this diagnostic. A residual-gas analyzer (RGA) instrument measures the gas content at various points in the system. The Digital Gamma Spectrometer instrument measures the radiological spectrum of the decaying gas isotopes. A final instrument controller was developed to interface to a PLC based Gas collection system.

In order to support the implosion velocity measurements an additional Gated X-ray Detector (GXD) diagnostic was tested and commissioned. This third GXD views the target through a slit contained in its snout and allows the other GXD diagnostics to be used for measuring the shape on the same shot.

In order to measure the implosion shape in a high neutron environment, Active Readout In A Neutron Environment (ARIANE) and Neutron Imaging (NI) diagnostics 
were commissioned. The controls for ARIANE, a fixed port gated x-ray imager, contain a neutron shielded camera and micro channel plate pulser with its neutron sensitive electronics located in the diagnostic mezzanine. The NI diagnostic is composed of two Spectral Instruments SI-1000 cameras located 20M from the target and provides neutron images of the DT hot spot for high yield shots.

The development and commissioning of these new or enhanced diagnostics in FY11 have provided meaningful insight that facilitates the optimization of the four key Ignition variables. In FY12 we will be adding three new diagnostics and enhancing four existing diagnostics in support the continuing optimization series of campaigns.

\section{Contact information}

Randy Shelton, shelton1@llnl.gov

Web site, lasers.llnl.gov 